\title{
Biomechanical evaluation of pin placement of external fixator in treating tranverse tibia fracture: Analysis on first and second cortex of cortical bone
}

\author{
Nur Amalina Zainudin ${ }^{a}$, Muhammad Hanif Ramlee ${ }^{a, b,}{ }^{,}$, Hadafi Fitri Mohd Latip ${ }^{b}$, Aizreena \\ Azaman ${ }^{b}$, Gan Hong Seng ${ }^{\text {c }}$, Evelyn Garcia-Nieto ${ }^{d}$, Mohammed Rafiq Abdul Kadir ${ }^{\text {a, b }}$ \\ ${ }^{a}$ Medical Devices and Technology Group (MEDITEG), School of Biomedical Engineering and Health Sciences, Faculty of Engineering, Universitti \\ Teknologi Malaysia, 81310 UTM Johor Bahru, Johor, Malaysia \\ Sports Innovation and Technology Centre (SITC), Institute of Humen Centered Engineering (iHumen), Universiti Teknologi Malaysia, 81310 UTM \\ Johor Bahru, Johor, Malaysia \\ c Universiti Kuala Lumpur, British Malaysian Institute, Jalan Sungai Pusu, 53100 Gombak, Selangor, Malaysia \\ ${ }^{d}$ Department of Mechanical Engineering, ETSII, Technical University of Madrid, 28006 Madrid, Spain
}

* Corresponding author: muhammad.hanif.ramlee@biomedical.utm.my

\section{Article history}

Received 11 August 2018

Revised 10 October 2018

Accepted 23 October 2018

Published Online 4 February 2018

\begin{abstract}
Biomechanical perspective of external fixator is one of the greatest factor to consider in successfully treating bone fracture. This is due to the fact that mechanical behavior of the structure can be analyzed and optimized in order to avoid mechanical failure, increase bone fracture healing rate and prevent pre-term screw loosening. There are three significant factors that affect the stability of external fixator which are the placement of pin at the bone, configuration and components of external fixator. These factors lead to one question: what is the optimum pin placement in which exerts optimum stability? To date, literature on above mentioned factors is limited. Therefore, we conducted a study to evaluate the uniplanar-unilateral external fixator for two different pin placement techniques in treating transverse tibia fracture via finite element method. The study was started off with the development of transverse tibia fracture using Mimics software. Computed tomography (CT) data image was utilized to develop three dimensional tibia bone followed by crafting fracture on the bone. Meanwhile, the external fixator was developed using SolidWork software. Both tibia bone and external fixator were meshed in 3-matic software with triangular mesh element. Simulation of this configuration was took place in a finite element software, Marc.Mentat software. A load of $400 \mathrm{~N}$ was applied to the proximal tibia bone in order to simulate stance phase of a gait cycle. From the findings, the pin placement at the second cortex of bone provided optimum stability in terms of stress distribution and displacement, which should be considered for better treatment for transverse tibia fracture. On the other hand, the pin placement at first cortex should be avoided to prevent many complications.
\end{abstract}

Keywords: Finite element analysis, pin placement, external fixator, transverse tibia fracture, stability

@ 2019 Penerbit UTM Press. All rights reserved

\section{INTRODUCTION}

In the worldwide, it is reported that $85 \%$ of extreme limb injuries come from lower limb cases(Fernandez et al., 2017). Lower limb region includes femur, patella, tibia, fibula, talus, hind foot, mid-foot and forefoot bone (van Niekerk et al., 2017). Accordingly, the tibia fracture is the most common bone fracture; contributes to at least $8.1 \%$ until $37 \%$ of lower limb injuries per annum (van Niekerk et al., 2017). This high percentage of injuries related to the lower limb region is due to the fact that the tibia bone is exposed to external load surrounding (Meleppuram \& Ibrahim, 2016). Besides, tibia injuries can be caused by an excessive internal force (compression, torsion, and shear) at the knee and ankle joint (Meleppuram \& Ibrahim, 2016).

In general, the tibia fracture can be divided into two categories; open and close fracture. The former is a fracture condition where some parts of tibia broken bone are exposed to outside tissue According to a study by Fernandez et. al (Fernandez et al., 2017), they found that the tibia fracture was occurred at least $10 \%$ in a year and
$40 \%$ of them were open fracture. Many medical surgeons experienced a lot of difficulties in treating open tibia fracture due to complications such as high rate of infections, as well as mal-union, non-union and mis-allignment of bone (Meleppuram \& Ibrahim, 2016). To avoid those complications during the treatment period, early fixation on the fracture is deeply necessary (Nambi et al., 2017. This is not only can prevent many complications to be occurred, but can also maximize the healing process of the broken bone of tibia (Nambi et al., 2017). One method to fix the open tibia fracture is by using an external fixator. The use of external fixator has been proven to be able to heal the broken bone within an appropriate time by alligning the tibia bone to its original position and minimizing the rate of infections (Elmedin et al., 2015).

The successful healing process of tibia fracture can be achieved in many ways. One of them is an optimum stability of the external fixator used in treating the fracture may increase the healing rate. As far as the authors are concerned, the external fixator is the most stable fixation compared with internal fixation such as screws, plates and 
wires (M. H. Ramlee et al.,2014a). The external fixator can prevent the movement of broken bone by fixating a number of pins at the desired position in tibia bone (Elmedin et al., 2015). The external fixator that is applied in this situation must indirectly preserve and protect damage of soft tissues while providing support to bone fracture from mobilization (Meng \& Zhou, 2016). Moreover, this system is a great alternative treatment for an easy applications due to the assembling of components in this system can maintain the stiffness and stability of the fracture in bone (Roseiro et al., 2014).

There are several configurations of external fixation:uniplanarunilateral, uniplanar-bilateral, biplanar, multiplanar and ring-type frame. The most common type of external fixator used by medical surgeons to treat a simple open tibia fracture is uniplanar-unilateral frame (Roseiro et al., 2014). However, pin of uniplanar-unilateral frame is often penetrated the bone. Some experts recommended to fix the pin until second cortex of bone while some of them recommended at first cortex (Prashanth et al., 2016). By considering these two situations, there is no argument found in the literature to justify the choices. From biomechanical point of view, stability of external fixator can alter the healing rate of broken bone. The external fixator that provides an optimum stability can minimize many complications as mentioned earlier (Prashanth et al., 2016).To our best knowledge, there is no study conducted yet to compare the above two choices. Therefore, this study was conducted with main aim to evaluate two different fixation techniques (pin passing through the second cortex versus pin locating at the first cortex) in order to treat transverse fracture via finite element method. The findings from this research would be valuable for medical surgeons to justify the choices in treating open tibia fracture, thus preventing more complications.

\section{MATERIALS AND METHOD}

\section{Three-dimensional modelling of tibia bone and fracture}

Three-dimensional (3D) model of tibia bone was reconstructed from Computed Tomography (CT) images data. The data was acquired from a $80 \mathrm{~kg}$ healthy male of 22 years old at Hospital Tunku Ampuan Afzan, Kuantan Pahang, Malaysia. A modelling software, Mimics (Materialise, Belgium), was used to reconstruct the 3D model of tibia in which consisted of cortical and cancellous bone (Oken et al., 2017; Ramlee et al., 2015). To differentiate between cortical and cancellous bone in Mimics software, a threshold value was set at 700(Ramlee et al., 2014a). Then, the segmentation and masking process were conducted to remove data noise. Some modifications were made where the bone's shape and size were in accordance with an anotomy book in mimicking the real geometrical conditions of tibia bone. The geometry of tibia bone was checked by a medical experts (Ramlee et al., 2014a). Once the 3D bone was completed, the next step was to develop a transverse fracture. This step was done by using Mimics software where a fracture gap of $4 \mathrm{~mm}$ was cut using cutting tools. The gap length was suggested from a study by Fagelberg et al. (Fågelberg et al., 2015) to simulate the tibia shaft transverse fracture. Finally the 3D model of tibia bone was triangular meshed with $3 \mathrm{~mm}$ size and was saved in STL format for next pre-processing step ( Ramlee et al., 2014a).

\section{Three-dimensional modelling of external fixator}

The uniplanar-unilateral external fixator was developed and designed by using a computer aided design (CAD) software, Solidworks (Dassault, USA). The modelling was started off by developing every single components such as pin, rod and clamp. The dimensions of those components were based on the commercial product of Synthes where the diameters of pin and rod were $5.5 \mathrm{~mm}$ and $11 \mathrm{~mm}$, respectively (Muhammad Hanif Ramlee et al., 2015).The length of rod was set at $170 \mathrm{~mm}$ (M. H. Ramlee, M. R. Kadir, M. R. Murali, \& T. Kamarul, 2014b). Later, all components of pin, rod and clamp were assembled into one single rigid body via similar CAD software. Then, the external fixator constructed was remeshed with triangular mesh element of $1.5 \mathrm{~mm}$ in size. Finally, the 3D model of external fixator was converted into STL file for futher pre-processing step.

\section{Virtual surgery}

Once the 3D model of tibia bone and external fixator were completed, the next step was to assemble both models. This step was conducted in Mimics software where the position of bone-to-rod was set to $40 \mathrm{~mm}$ distance (Sternick et al., 2012). In this study, there were two different fixation techniques i.e. Fixation 1 that assembled the external fixator to be fixated through the second cortex of tibia bone and Fixation 2 that only considered pin penetration at first cortex, as shown in Fig. 1. Both external fixation techniques were consisted of four pins, four clamps and one connecting rod. Once the position of the external fixator was set, a virtual surgery was conducted to fix the fixator onto the bone by applying 'create manifold assembly' tool in the Mimics software. Due to manifold assembly, some modifications were performed to modify the non-uniform triangular mesh element at the pin-bone interface into uniform size of element. This was done in 3-Matic software. a)

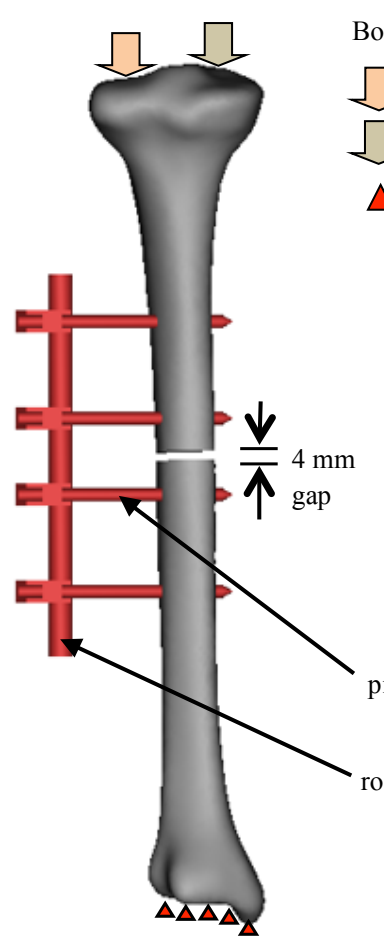

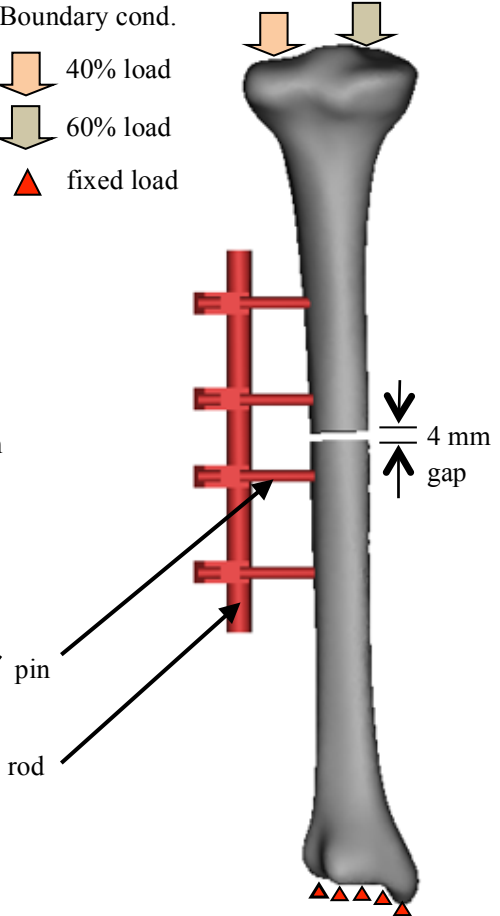

b)
Fig. 1 The fixation techniques for a) Fixation 1 b) Fixation 2.

\section{Finite element analysis}

All STL files of the tibia bone and external fixator were imported into a finite element software, Marc.Mentat (MSC.Software, USA). The files were then converted into $3 \mathrm{D}$ model of linear first-order tetrahedral elements (Abd Rahman et al., 2008). For material properties, the 3D tibia bone of cortical and cancellous were set at 16.2 $\mathrm{GPa}$ and $480 \mathrm{MPa}$ modulus, respectively (Pitkin et al., 2007). Meanwhile their Poisson's ratios were 0.3 and 0.26 , respectively (M. H. Ramlee et al., 2014a). As for the external fixator, a stainless material was used to simulate it where the material properties was set at 200,000 $\mathrm{MPa}$. All models were assigned with isotropic and homogeneous material properties. A convergence analysis was performed in our previous study (Ramlee et al., 2014a) using hrefinement method where the optimum mesh size for bone was $3 \mathrm{~mm}$ and the external fixator was $1.5 \mathrm{~mm}$. A validation of the finite element model was conducted in the previous reported literature. The validation results showed that all four selected points at tibia bone were similar to that experimental findings (Ramlee et al., 2018). The previous findings showed that the trend and profile of the graph were the same for both the finite element results and experimental data. 


\section{Boundary conditions}

In this study, we simulated a stance phase condition to evaluate the fixation techniques. According to a study, $50 \%$ of the body weight was applied to one part of lower limb of human (Oken et al., 2017). Since the body weight of patient was $80 \mathrm{~kg}$, therefore a $400 \mathrm{~N}$ load was applied to the proximal tibia bone, where $60 \%$ of the load at the medial curviture and another $40 \%$ at the lateral curviture as shown in the Fig. 1 (Raja Izaham et al., 2012). The axial compression force was normally applied by many researchers to mimic the weight bearing condition.

\section{RESULTS AND DISCUSSION}

\section{Equivalent Von Misses Stress}

Fig. 2 shows the contour plot of equivalent von Mises stress of tibia bone for both fixation techniques during a stance phase of a gait cycle. From the results, in general, the high stress was found at the pin-bone interface for both fixations where the highest value was specifically located at the distal pin-bone. Undoubtedly, the high stress at this surrounding tissue was similar to previous study by others where this could not be avoided when using an external fixator (Brianza et al., 2011; Donaldson et al., 2012). As comparing both fixation techniques, the Fixation 2 experienced higher stress $(21.41$ $\mathrm{MPa})$ than Fixation $1(15.20 \mathrm{MPa})$ with $34 \%$ difference in value. Due to the high stress for Fixation 2, this was a main reason on why medical experts did not allow patients to experience full weight bearing during a period of treatment (Ansah \& Sella, 2000; Dlimi et al., 2011; Harris et al., 2008). Nevertheless, this high value of stress at the critical local point of pin-bone interface wasnot exceed the ultimate strength of bone (193 MPa) (Pinner \& Sangeorzan, 2001). From the Fig. 2, it could be observed that high stress was illustrated at approximately $10 \mathrm{~cm}$ above tibia malleolus for the Fixation 2 compared to the Fixation 1. This high stress at surrounding tissues might lead to secondary fracture if patients were allowed to experience full gait condition. Based on these findings, it was suggested that medical surgeons and patients should take extra care if Fixation 2 was applied to treat transverse tibia fracture, in order to avoid any complications during early medical interventions.

Fig. 3 shows the contour plot of equivalent von Mises stress of external fixator for both fixation techniques. Between both fixations, a small difference $(4.5 \%)$ was found at the second pin of external fixator. The higher stress was experienced in the Fixation 2 (129.55 $\mathrm{MPa})$ compared to the Fixation $1(123.83 \mathrm{MPa})$. To be noted, the uniform distribution of stress was illustrated at the rod of external fixator for both techniques. There was no significant difference in Fixation 1 and Fixation in terms of stress value at this particular point of rod. From these findings, both fixation techniques were safe to be used in treating transverse tibia fracture in terms of biomechanical aspect due to the fact that the stresses did not exceed the ultimate strength of that particular material (800-900 MPa) (Gorsse \& Miracle, 2003; M.H. Ramlee et al., 2018). As far as the authors were concerned, the use of external fixator was favourable option to allow weight bearing forces to be distributed from the upper to lower patient's body through the pin and rod. Therefore, minimal force distribution would impose to the broken bone. From this study, we simulated the global stress value at the external fixator where we found that higher stress was located at pin-bone interface. For the future research, we were highly recommended that other researchers could focus on the local stress at the clamps. This was due to the fact that high stress at the clamps could allow pin and rod loosening during the weight bearing, thus affecting the stability of the external fixator. Other than that, stress shielding at the pin-bone interface could be evaluated further in the next study where this could predict the pin loosening.

\section{Displacement}

Global displacements of the two different techniques were illustrated in Figure 4. In general, the global displacement for Fixation 1 showed a significant difference at the proximal tibia bone as compared with Fixation 2. The proximal tibia bone of the Fixation 1 experienced at least $3.21 \mathrm{~mm}$ of displacement, while for the Fixation 2 was $1.42 \mathrm{~mm}$. These values demonstrated at least $77.3 \%$ of difference. For the external fixator alone, the Fixation 1 and Fixation 2 showed at least $1.67 \mathrm{~mm}$ and $1.20 \mathrm{~mm}$ of displacement, respetively, where demonstrated at least $32.8 \%$ of difference. This small value of displacement at the external fixator concluded that both techniques were safe to be used, however medical surgeons should consider the movement of tibia bone to avoid complications. Based on the high displacement at the proximal tibia for Fixation 1, it should be avoided since the healing process of a broken bone would take place only if small displacement or micromovement was appeared. Nevertheless, future research should be conducted to prove this claim.
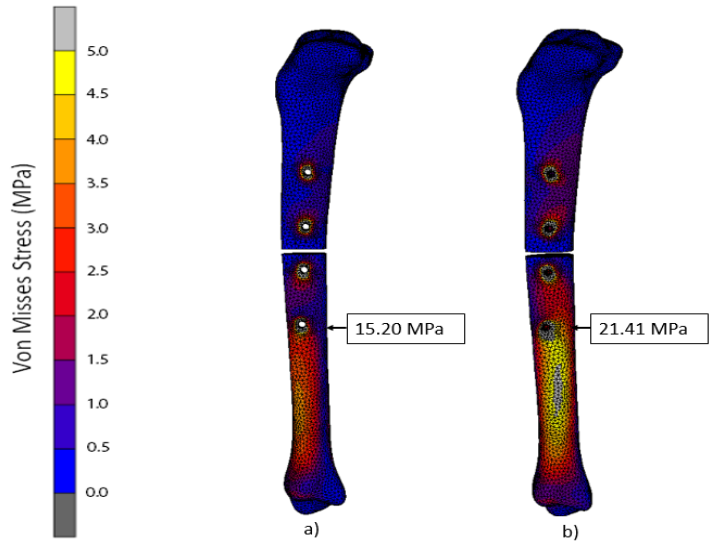

Von Mises Stress (MPa)

Fig. 2 Stress contour plot of tibia bone for a) Fixation 1 b) Fixation 2.
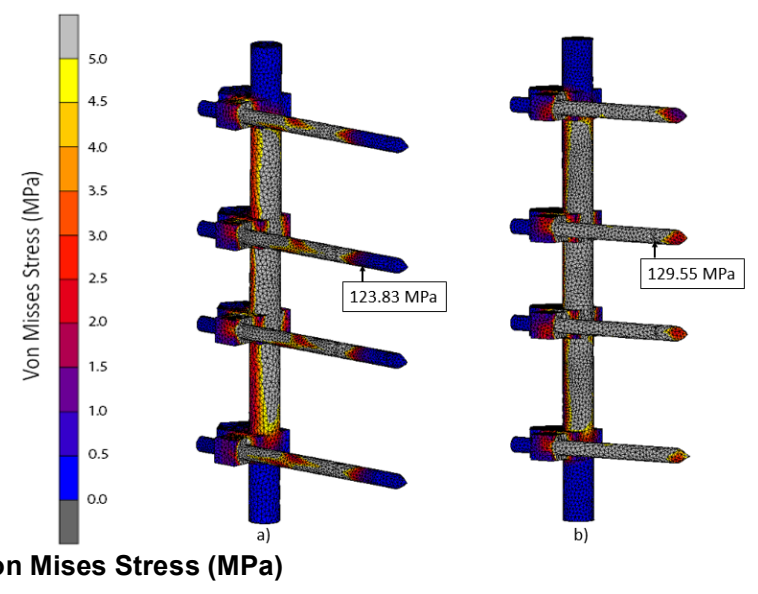

Fig. 3 Stress contour plot of external fixator fora) Fixation 1 b) Fixation 2 .
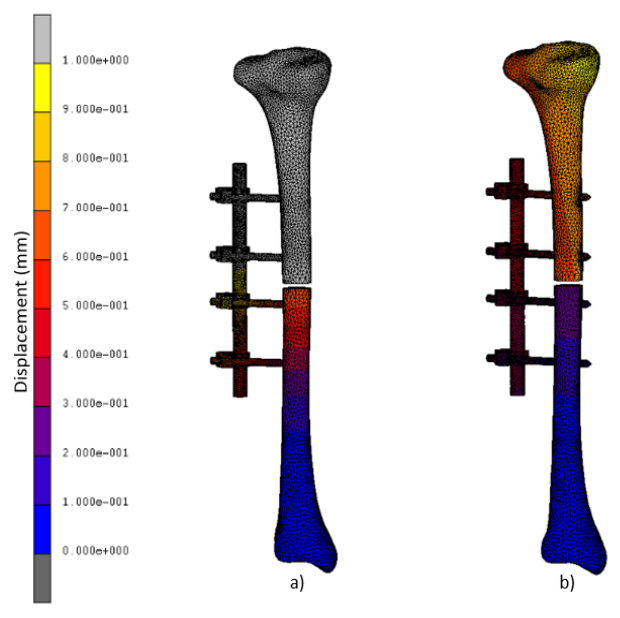

Fig. 4 Displacement contour plot for a) Fixation 1 b) Fixation 2. 
Fig. 5 shows the global mapping condition between deformed and original model for both Fixation 1 and 2. Fixation 1 has more obvious deformation than Fixation 2. Based on this findings, it was not suggested to use the first technique which was the Fixation 1 in treating tranverse tibia fracture.

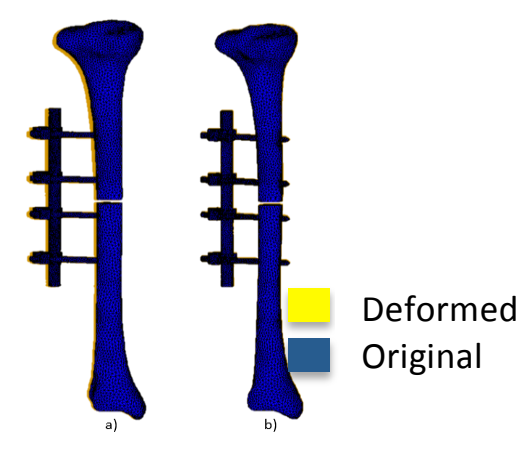

Fig. 5 Mapping of original and deformed configuration for a) Fixation 1 b) Fixation 2 .

The use of external fixator in treating tibia fracture is considered as favourable option as compared to other medical devices. The ability of the external fixator in providing minimal invasive procedure giving adequate stability and promoting less infection proved that this medical device is opted for the best choic among others (Pinner \& Sangeorzan, 2001; Seibert et al., 2003). Moreover, the high stability of the external fixator has been taken into consideration, giving that it could hasten soft tissue healing during the medical intervention period (Chandran et al., 2006; Seibert et al., 2003). However, before the successful outcome can be delivered, a proper step and procedure should be conducted properly by medical experts upon the surgery process. One of the step is by choosing the correct fixation techniques. As demonstrated in this study, there were two different techniques have been analysed to evaluate the best external fixator. Based on the results, it was suggested that the Fixation 2 as the good fixation technique where the pin was penetrated until the second cortex of bone. In terms of biomechanical aspect, the technique of Fixation 2 could provide adequate stability to the broken bone and avoid many complications such as mal-union and mis-allignment. On the other hand, if the Fixation 1 was used, above complications might appear in treating the patients. It should be noticed that inability to provide a stable construction might cause secondary fracture at the pin-bone interface (Inokuchi et al., 1996; Vasquez et al., 2003).

As far as the authors concerned, biomechanical studies related to the external fixator for lower limb of human were very limited. Therefore, this study was conducted to justify the medical surgeon's choices in treating lower limb injuries. In addition, these findings were expected to help medical experts and engineers to choose suitable configurations and techniques for a specific pathological problem of tranverse tibia bone. Other than these findings, the studies that have been conducted were only on the external fixator for the horse, ankle dislocations and pilon fractures of human (M.H. Ramlee, Kadir, \& Harun, 2014; M. H. Ramlee, M. R. A. Kadir, M. R. Murali, \& T. Kamarul, 2014; M.H. Ramlee et al., 2018). In accordance with previous studies, this work also reported similar high stress at the pin-bone interface where this situation could not be avoided since the material for external fixator wasis higher than of bone.

In this study, there were several assumptions and limitations to be considered since the assumptions could not be simply avoided when dealing with finite element simulation. Firstly, the material properties for all models of cortical and cancellous bone were assigned with linear, isotropic and homogeneous characteristics, thus all other factors that might alter the predicted results were excluded. Although these characteristics wereare unreal, nevertheless these assumptions were normally used by the others and became acceptable as demonstrated by many researchers (Benli et al., 2008; Brianza et al., 2011; Ezquerro et al., 2007; Izaham et al., 2012). In comparing isotropic and orthotropic characteristics, a study has been conducted by others in which there was only small difference $(1.2 \%)$ in terms of maximum equivalent von Mises stress and the maximum nodal displacement (Peng et al., 2006). Thus it was recommended that the future research to consider the usage of greyscale value of CT images in mimicking real properties of the cortical and cancellous bone. Other than that, the use of inhomogeneous properties should be considered in later studies. Second limitation in this study was the limitation of region of interest (ROI) of the bone to tibia only due to computer resources. Nevertheless the future study should consider the whole lower limb in order to provide optimum results. Another limitation in this study was the stance phase loading. In this work, the stance phase of a gait cycle was only simulated in statics condition due to the fact that medical surgeons would not allow patients to experience full weight bearing after the surgery. The best practise in simulating the gait cycle was by applying all the phases. The next limitation was the axial load applied at the proximal tibia bone. The value of $50 \%$ of patient's weight was taken from previous study in which the load from Achilles tendon force was only simulated during the stance phase condition (Simkin, 1982). Meanwhile, other forces from other muscles such as longus (peroneus, flexor digitorum, flexor halluces) and peroneus brevis were not considered in this study due to low magnitude of forces.

For the development of tibia bone, it was based on the CT images from a healthy person where the fracture was constructed later on. This process was another limitation of this work. It was strongly suggested that patient-specific condition should be used to model transverse tibia fracture in the future. Furthermore, the development of 3D tibia model was also simplified by applying Hounsfield unit (HU) at 700 to differentiate cortical and cancellous bone. To date, a technique called as micromechanics-based determination of voxelspecific Young's modulus and Poisson's ratio should be considered in 3D model tibia bone. For the results, von Mises stress was simluated for evaluating the failure criterion of bone (Fig. 2). There was no issue for stainless steel external fixator (Fig. 3) since the characteristics of that particular material were isotropic, linear and homogeneous. However, the bone itself was not steel due to the characteristics of bone failure could be known as water-induced ductile and followed by rupture of collagen crosslinks (Fritsch et al., 2009). Therefore, it was suggested that the failure characteristics to be analysed via MohrCoulomb failure criterion. Nevertheless, our results on von Mises stress were still acceptable after considering above limitations and assumptions as demonstrated by other scholars (Chen et al., 2012; Cheung et al., 2006; Haraguchi et al., 2009; Izaham et al., 2012; Kim et al., 2011).

Other limitation in this study was the design of external fixator. The development of external fixator was not considered the pin thread, rod thread and clamp thread. The external fixator was only developed by considering one single body which was another simplication due to computer resources. Future studies should develop a very detail of external fixator in giving off better findings.

\section{CONCLUSION}

This study has been successfully conducted where the main aim was to evaluate the fixation techniques of external fixator to treat tranverse tibia fracture. Based on the obtained results, the Fixation 2 was considered as the best technique where the pin placement was located and penetrated until the second cortex of cortical bone to allow full weight bearing for patients. On the other hand, if medical surgeons applied the Fixation 1, extra care should be considered in allowing patients to stand alone due to high stress and displacement at both bone and external fixator. Therefore, to allow full weight bearing for patients, it was suggested that the Fixation 2 should be used.

\section{ACKNOWLEDGEMENT}

Special thanks to the Director of Universiti Malaya Medical Centre (UMMC), Prof. Dr. Tunku Kamarul for his efforts and willingness to properly check the geometrical size of tibia bone model. Thank you to all students and staffs from the School of Biomedical Engineering and Health Sciences, Universiti Teknologi 
Malaysia (UTM) especially who are from Medical Devices and Technology Group (MEDITEG) for their endless supports in conducting this recent study. Thanks again to MEDITEG for providing special workspace and equipments to simulate the finite element model. This study was financially supported by UTM Research Grant under Tier 1 scheme (Q.J130000.2745.20H26 and Q.J130000.2545.20H20).

\section{REFERENCES}

Abd Rahman, H. S., Abu Osman, N. A., Wan Abas, W. A. B., Tunku Ahmad T. S., Ng, E. S. (2008). Validation of finite element analysis for a new external finger fixator to correct flexion deformity - a preliminary result. In N. A. Abu Osman, F. Ibrahim, W. A. B. Wan Abas, H. S. Abdul Rahman, H.-N. Ting (Eds.), 4th Kuala Lumpur International Conference on Biomedical Engineering 2008: BIOMED 2008 25-28 June 2008 Kuala Lumpur, Malaysia (pp. 465-468). Berlin, Heidelberg: Springer Berlin Heidelberg.

Ansah, M., Sella, E. J. (2000). Treatment of complete open medial subtalar dislocation with an external fixateur: A case report. Foot and Ankle Surgery, 6, 179-184.

Benli, S., Aksoy, S., Havitcioglu, H., Kucuk, M. (2008). Evaluation of bone plate with low-stiffness material in terms of stress distribution. Journal of Biomechanics, 41, 3229-3235.

Brianza, S., Brighenti, V., Lansdowne, J. L., Schwieger, K., Boure, L. (2011) Finite element analysis of a novel pin-sleeve system for external fixation of distal limb fractures in horses. The Veterinary Journal, 190, 260-267.

Chandran, P., Puttaswamaiah, R., Dhillon, M. S., Gill, S. S. (2006) Management of complex open fracture injuries of the midfoot with external fixation. The Journal of Foot and Ankle Surgery, 5(45), 308-315.

Chen, D. W., Lin, C. L., Hu, C. C., Wu, J. W., Lee, M. S. (2012). Finite element analysis of different repair methods of Vancouver B1 periprosthetic fractures after total hip arthroplasty. INJURY, 43(7), 10611065.

Cheung, J. T.-M., Zhang, M., An, K. N. (2006). Effect of Achilles tendon loading on plantar fascia tension in the standing foot. Clinical Biomechanics, 21, 194-203.

Dlimi, F., Mahfoud, M., Berrada, M. S., Bardouni, A. E., Yaacoubi, M. E. (2011). Open medial ankle dislocation without associated fracture : A case report. Foot and Ankle Surgery, 17, e55-e57.

Donaldson, F. E., Pankaj, P., Simpson, A. H. R. W. (2012). Bone properties affect loosening of half-pin external fixators at the pin-bone interface. Injury, 43, 1764-1770.

Elmedin, M., Vahid, A., Nedim, P., Nedžad, R. (2015). Finite Element Analysis and Experimental Testing of Stiffness of the Sarafix External Fixator. Procedia Engineering, 100, 1598-1607.

Ezquerro, F., Jimenez, S., Perez, A., Prado, M., de Diego, G., Simon, A. (2007). The influence of wire positioning upon the initial stability of scaphoid fractures fixed using Kirschner wires: A finite element study. Medical Engineering and Physics, 29, 652-660.

Fågelberg, E., Grassi, L., Aspenberg, P., Isaksson, H. (2015). Surgical widening of a stress fracture decreases local strains sufficiently to enable healing in a computational model. International Biomechanics, 2(1), 12 21.

Fernandez, M. A., Nanchahal, J., Costa, M. L. (2017). Open tibial fractures. Orthopaedics and Trauma, 31(2), 125-132.

Fritsch, A., Hellmich, C., Dormieux, L. (2009). Ductile sliding between mineral crystals followed by rupture of collagen crosslinks: Experimentally supported micromechanical explanation of bone strength. Journal of Theoretical Biology, 260, 230-252.

Gorsse, S., Miracle, D. B. (2003). Mechanical properties of Ti-6Al-4V/TiB composites with randomly oriented and aligned TiB reinforcement. Acta Materialia, 51, 2427-2442.

Haraguchi, N., Armiger, R. S., Myerson, M. S., Campbell, J. T., Chao, E. Y. (2009). Prediction of three-dimensional contact stress and ligament tension in the ankle during stance determined from computational modeling. Foot and Ankle International, 30, 177-185.

Harris, J., Huffman, L., Suk, M. (2008). Lateral Peritalar Dislocation: A Case Report. The Journal of Foot and Ankle Surgery, 47(1), 56-59.

Inokuchi, S., Hashimoto, T., Usami, N., Ogawa, K. (1996). Subtalar dislocation of the foot. Foot, 6, 168-174.

Izaham, R. M. A. R., Kadir, M. R. A., Rashid, A. H. A., Hossain, M. G., Kamarul, T. (2012). Finite element analysis of Puddu and Tomofix plate fixation for open wedge high tibial osteotomy. INJURY, 43, 898-902.

Kim, H.-J., Kim, S.-H., Chang, S.-H. (2011). Bio-mechanical analysis of fractures tibia with composite bone plates according to the diaphyseal oblique fracture angle. Composites Part B: Engineering, 42, 666-674.
Meleppuram, J. J., Ibrahim, S. (2016). Experience in fixation of infected nonunion tibia by Ilizarov technique - a retrospective study of 42 cases. Revista Brasileira de Ortopedia (English Edition), 52(6), 670-675.

Meng, Y.-C., Zhou, X.-H. (2016). External fixation versus open reduction and internal fixation for tibial pilon fractures: A meta-analysis based on observational studies. Chinese Journal of Traumatology, 19(5), 278-282.

Nambi, G. I., Salunke, A. A., Thirumalaisamy, S. G., Babu, V. L., Baskaran, K., Janarthanan, T. (2017). Single stage management of Gustilo type III A/B tibia fractures: Fixed with nail \& covered with fasciocutaneous flap. Chin J Traumatol, 20(2), 99-102.

Oken, O. F., Yildirim, A. O., Asilturk, M. (2017). Finite element analysis of the stability of AO/OTA 43-C1 type distal tibial fractures treated with distal tibia medial anatomic plate versus anterolateral anatomic plate. Acta Orthopaedica et Traumatologica Turcica, 51(5):404-408

Peng, L., Bai, J., Zeng, X., Zhou, Y. (2006). Comparison of isotropic and orthotropic material property assignments on femoral finite element models under two loading conditions. Medical Engineering \& Physics, $28,227-233$.

Pinner, S. J., Sangeorzan, B. J. (2001). Fractures of the tarsal bones. Orthopaedic Clinical of North America, 32, 21-33.

Pitkin, M., Shukeylo, Y., Gritsanov, A. (2007). Mathematical modeling of fixation of a bone fragment in a new Double-needle external fixator compared to hoffmann II fixator. Ser Biomech, 23(1), 96-103.

Prashanth, D. P. S., Singh, P. S. N., Singh, P. A. M., Mukherjee, D. S., Pertin, D. T., Pakhrin, D. V. (2016). A comparative study of uniplanar unilateral external fixationversus locking plate as external fixation in the definitive management of open fractures of tibial diaphysis in adults. IOSR Journal of Dental and Medical Sciences, 15(08), 50-52.

Raja Izaham, R. M., Abdul Kadir, M. R., Abdul Rashid, A. H., Hossain, M. G., Kamarul, T. (2012). Finite element analysis of Puddu and Tomofix plate fixation for open wedge high tibial osteotomy. Injury, 43(6), 898-902

Ramlee, M. H., Kadir, M. R., Murali, M. R., Kamarul, T. (2014a). Biomechanical evaluation of two commonly used external fixators in the treatment of open subtalar dislocation - a finite element analysis. Medical Engineering \& Physics, 36(10), 1358-1366.

Ramlee, M. H., Kadir, M. R., Murali, M. R., Kamarul, T. (2014b). Finite element analysis of three commonly used external fixation devices for treating Type III pilon fractures. Medical Engineering \& Physics, 36(10), 1322-1330.

Ramlee, M. H., Kadir, M. R. A., Harun, H. (2014). Three-dimensional modelling and finite element analysis of an ankle external fixator. Advanced Materials Research, 845, 183-188.

Ramlee, M. H., Kadir, M. R. A., Murali, M. R., Kamarul, T. (2014). Biomechanical evaluation of two commonly used external fixators in the treatment of open subtalar dislocation- A finite element analysis. Medical Engineering \& Physics, In press.

Ramlee, M. H., Rafiq Abdul Kadir, M., Harun, H. (2015). Three-dimensional modeling and analysis of a human ankle joint. 2013 IEEE Student Conference on Research and Development. 16-17 Dec. Putrajaya: IEEE.

Ramlee, M. H., Sulong, M. A., Garcia-Nieto, E., Penaranda, D. A., Felip, A. R., Abdul Kadir, M. R. (2018). Biomechanical features of six design of the delta external fixator for treating Pilon fracture: A finite element study. Medical \& Biological Engineering \& Computing, 56(10), 1925-1938.

Roseiro, L. M., Neto, M. A., Amaro, A., Leal, R. P., Samarra, M. C. (2014). External fixator configurations in tibia fractures: 1D optimization and 3D analysis comparison. Computer Methods and Programs in Biomedicine, 113(1), 360-370.

Seibert, F. J., Fankhauser, F., Elliot, B., Stockenhuber, N., Peicha, G. (2003). External fixation in trauma of the foot and ankle. Clinical Podiatric Medicine and Surgery, 20(139-130).

Simkin, A. (1982). Structural analysis of the human foot in standing posture. (Ph.D.), Tel Aviv University, Tel Aviv.

Sternick, M. B., Dallacosta, D., Bento, D. Á., do Reis, M. L. (2012). Relationship between Rigidity of External Fixator and Number of Pins: Computer Analysis Using Finite Elements. Revista Brasileira de Ortopedia (English Edition), 47(5), 646-650.

van Niekerk, A. H., Birkholtz, F. F., de Lange, P., Tetsworth, K., Hohmann, E. (2017). Circular external fixation and cemented PMMA spacers for the treatment of complex tibial fractures and infected nonunions with segmental bone loss. Journal of Orthopaedic Surgery (Hong Kong), 25(2), 2309499017716242.

Vasquez, A. A., Pedersen, H. L., Lidgren, L., Taylor, M. (2003). Finite element analysis of the initial stability of ankle arthodesis with internal fixation: flat cut versus intact joint contours. Clinical Biomechanics, 18, 244-253. 